\title{
VLF Trimpi modelling on the path NWC-Dunedin using both finite element and 3D Born modelling
}

\author{
D. Nunn ${ }^{\mathrm{a}, *}$, K. Baba ${ }^{\mathrm{b}}$ M. Hayakawa ${ }^{\mathrm{c}}$ \\ ${ }^{a}$ Department of Electronics and Computer Science, Southampton University, U.K. \\ ${ }^{\mathrm{b}}$ Chubu University, Japan \\ ${ }^{\mathrm{c}}$ University of Electro Communications, Tokyo, Japan
}

Received 22 April 1998; received in revised form 11 August 1998; accepted 25 August 1998

\begin{abstract}
This paper investigates the numerical modelling of VLF Trimpis, produced by a D region inhomogeneity on the great circle path. Two different codes are used to model Trimpis on the path NWC-Dunedin. The first is a 2D Finite Element Method Code (FEM), whose solutions are rigorous and valid in the strong scattering or non-Born limit. The second code is a $3 \mathrm{D}$ model that invokes the Born approximation. The predicted Trimpis from these codes compare very closely, thus confirming the validity of both models. The modal scattering matrices for both codes are analysed in some detail and are found to have a comparable structure. They indicate strong scattering between the dominant TM modes. Analysis of the scattering matrix from the FEM code shows that departure from linear Born behaviour occurs when the inhomogeneity has a horizontal scale size of about $100 \mathrm{~km}$ and a maximum electron density enhancement at $75 \mathrm{~km}$ altitude of about 6 electrons. (C) 1998 Elsevier Science Ltd. All rights reserved.
\end{abstract}

\section{Introduction}

The Trimpi phenomenon is a well known geophysical problem in VLF wave propagation that has been extensively studied both experimentally, and theoretically. Full reviews of this topic may be found in Strangeways (1996), Smith (1996) and also in Nunn (1997). Trimpis comprise transient perturbations in amplitude and phase of subionospheric VLF signals. These transients are typically of quite a small amplitude, $0.3 \mathrm{~dB}$ and 5 degrees of phase, but they may vary from $1-30$ degrees in phase, and $0.1-$ $3 \mathrm{~dB}$ in amplitude. Trimpis are observed with a full range of scatter phase, but the most common type is the $(-+)$ Trimpi with negative amplitude and positive phase perturbations. The Trimpi onset time is short $(\sim 100 \mathrm{~ms})$, and the decay time typically is of order $100 \mathrm{~s}$.

The so-called 'Classic Trimpis' are believed to be due to lightning whistlers, which interact at the equator with radiation belt $\mathrm{keV}$ electrons, causing them to precipitate into the $\mathrm{D}$ region of the nighttime ionosphere, giving

*Corresponding author. Tel.: +44 1703 592969; fax: +44 1703 593045; e-mail: d.nunn@ecs.soton.ac.uk localised perturbation patches of enhanced electron density (Helliwell, 1973). These Localised Ionisation Enhancements, or LIEs, occupy a height range of 65$85 \mathrm{~km}$, with horizontal dimensions of order $40-150 \mathrm{~km}$ (Dowden and Adams, 1989). The vertical dimensions of a LIE can be inferred from the computed energy spectrum of electrons precipitated by a lightning whistler (Lev-Tov et al., 1996). The horizontal scale size of a LIE must be determined by the average size of a whistler duct at the equator. It is clear though that LIEs with a horizontal scale size $<50 \mathrm{~km}$ are too small to create an observable Trimpi. These LIEs scatter subionospheric VLF signals, and the scattered field gives rise to the VLF phase and amplitude perturbation, or Trimpi.

The general consensus now is that some observations of Trimpis are not due to energetic electron precipitation from the magnetosphere, but are a result of scattering from $\mathrm{D}$ region inhomogeneities caused directly by the electric field or EM radiation field of the lightning discharge. In this connection a recent topic of interest is that of Sprites, or cloud to ionosphere discharges (Dowden et al. (1996); Strangeways (1996); Schonland and Craib (1927)). These are believed to consist of narrow columns of enhanced electron density with a value 
believed to be at least two orders of magnitude greater than that of the classic LIE; Sprites may cause 'fast early' Trimpis or RORDs. (Inan et al., 1988; Dowden et al. (1994)). Inan (1990) also discovered that direct heating due to a powerful VLF transmitter or lightning can also cause a localised $\mathrm{D}$ region LIE of elevated temperature and collision frequency, capable of giving rise to Trimpis. Indeed a recent paper by Molchanov et al. (1998), hypothesises that a significant fraction of Trimpis are due to electron density $\mathrm{D}$ region enhancements caused directly by the lightning discharge and not by whistler precipitation.

In this paper we will only be concerned with the 'classic' VLF Trimpi, but the FEM and 3D Born codes used here may be readily applied to the problem of scattering from small scale strong structures such as Sprite columns, as well as from heated patches. There is clearly a need for accurate modelling of the mechanism producing Trimpis. Apart from advancing the theory of scattering in ionospheric plasma, modelling can help to resolve the vexed question of the origins of the causative D region inhomogeneities. It will also enable us to use Trimpi observations, from multiple transmitters and multiple receivers, to solve the 'inverse problem' and map charged particle precipitation into the ionosphere. Precipitation is important as it represents a substantial energy flux from space into the Earth-ionosphere system. This energy flux is believed to be somewhat increased by higher levels of lightning activity due to global warming (Williams, 1992), and also by ELF/VLF radiation from power line systems and industrial plant, the so called Power Line Harmonic radiation (PLHR) (Molchanov and Parrot 1995; Bullough, 1995).

We shall model great circle Trimpis observed on the path from the $22.3 \mathrm{kHz}$ VLF transmitter NWC in NW Australia to Dunedin in New Zealand (Dowden and Adams 1989, 1990). Note that in June 1992 the frequency of NWC changed to $19.8 \mathrm{kHz}$, but we shall use the old frequency. This is a mixed land-sea path, $5.74 \mathrm{Mm}$ in length. The modelling will use two distinct techniques. The first is FEM, or Finite Element Method, applied by Baba and Hayakawa (1995, 1996), in a 2D, rigorously non-Born approach (i.e., strong scattering). The second is due to Nunn (1997), and embodies a fully 3D, linear scattering Born formalism.

Our task is to compare the results from these two codes closely using scattering scenarios that are as close as possible. This will serve to validate both theoretical approaches. When the LIE is very large or strong, the incident zero order wavefield will have a skin depth of the order or less than the patch size, and will be progressively excluded from the patch, resulting in a breakdown of the Born approximation. By careful analysis of results from the FEM code, we will determine the range of LIE sizes and strengths for which the Born approximation is valid. We shall also examine in detail the modal scattering matr- ices and assess the importance of scattering from one mode to another.

\section{Previous theoretical and numerical approaches to modelling}

Progress towards accurate numerical modelling of the Trimpi phenomenon has been surprisingly slow. Poulsen et al. (1993) adopted a linear scattering (Born) approach and used an expression due to Wait $(1964,1964 a)$ which assumes that intermodal scattering is negligible and that the spatial scale of the patch is much greater than a wavelength. Neither condition is satisfied, and our results will show that intermodal scattering is always significant, in full agreement with theoretical and analytic studies in Wait (1991, 1995).

More recently, Baba and Hayakawa $(1995,1996)$ have used the finite element method (FEM) to attack the Trimpi problem. They use Galerkin's FEM technique which is classically used for solving complex EM field problems in difficult geometries (McDonald and Wexler, 1972). In view of the complexity of the problem, their treatment is $2 \mathrm{D}$. The Earth is assumed a perfect conductor, and the ionosphere isotropic $(B o=0)$, with realistic electron density and collision frequency profiles. Currently Earth curvature is neglected. Important results have been obtained with this code, since it is the only approach so far to this problem that does not invoke the Born approximation.

The method adopted in Nunn (1997) is the opposite of the above. The model is fully $3 \mathrm{D}$ with an anisotropic ionosphere, and Earth curvature. Of necessity, however, it invokes the weak scattering or Born approximation. At each point in the LIE an effective source current $J_{\text {eff }}(r)$ may be defined by

$J_{\mathrm{eff}}(r)=\frac{j k^{2}}{\omega \mu_{0}} X^{\prime}(r) E_{0}(r)=\sigma^{\prime} E_{0}$

where $X^{\prime}(r)$ is the perturbation in susceptibility tensor, and $E_{0}(r)$ is the zero order incident field (Simonich and Yeh, 1972). The scattered field $E^{\prime}(r)$ is sourced upon the current distribution $J_{\text {eff }}(r)$ located within the LIE. The code of Nunn (1997) makes the approximation that in the conductivity tensor $\sigma^{\prime}$ only the $z z$ component is nonzero. This is equivalent to ignoring the horizontal components of source currents in the LIE. Although these may be sizeable for an anisotropic ionosphere, they will only weakly excite the TM modes which will be observed at the receiver. Numerical experimentation showed that this approximation gives an overall error in calculated Trimpis of $<10 \%$.

It now remains to deal with the propagation problem. Nunn (1997) used Wait's modal theory and its computer implementation, the National Ocean Systems Centre 
(NOSC) VLF propagation facility MODEFNDR (Morfitt and Shellman, 1976). For short ranges $<300$ $\mathrm{km}$, modal theory is not valid if only 12 modes are used. In this case a full wave approach is obligatory, though this remains to be implemented. In recent work by R. Yeo (Clilverd et al., 1998), propagation is handled by the NOSC LWPC Long Wave Propagation Capability (Ferguson and Snyder, 1990), which incorporates global scale conductivity and ionospheric maps. This code also uses the full $\sigma^{\prime}$ tensor.

In a recent short review letter (Baba et al., 1998), an initial comparison of the FEM and 3D Born codes was made. The 3D Born code was used with the ambient ionospheric magnetic field turned off. The two codes were used to model Trimpis on the path NWC-Dunedin as here, the main physical difference between the two codes being the absence of Earth curvature in the FEM code. When the wavefield incident on the LIE consisted of the dominant TM mode, the two codes gave very good agreement indeed. This paper will compare the FEM code with the anisotropic 3D Born code, and will greatly extend the investigation of the Born approximation and modal scattering matrices.

\section{The numerical modelling}

\subsection{The FEM code}

The FEM code has two independent spatial coordinates, $x$ along the direction of propagation and $z$ vertically. The enhancement of electron density in the LIE, $\delta \mathrm{N}_{\mathrm{e}}(x, z)$, is assumed to be a Gaussian function as follows:

$\delta N_{\mathrm{e}}(x, z)=\delta N_{\mathrm{e}}^{0} \exp \left\{-\left(\frac{x-x_{0}}{S_{x}}\right)^{2}-\left(\frac{z-z_{0}}{S_{z}}\right)^{2}\right\}$

The average patch height is taken to be $z_{0}=75 \mathrm{~km}$, a figure which coincides with the level of maximum scattering (Nunn, 1997), and also agrees will computations of electron density enhancements due to whistler precipitation (Inan and Carpenter, 1987). A Gaussian half width of $S_{z}=10 \mathrm{~km}$ was generally used. The horizontal scale of the LIE $S_{x}$ is not well known and was varied from $10-100 \mathrm{~km}$. The quantity $x_{0}$ is the centre of the patch in the $x$ dimension. The FEM code employs an isotropic ionosphere with a typical exponential nighttime electron density profile given by (Reagan et al., 1982)

$N_{0}(z)=(30.24) \exp \{0.24(z-H)\} \quad$ electrons/cc

where $H=87 \mathrm{~km}$ and $z$ is in $\mathrm{km}$. The assumed collision frequency profile is again the standard one:

$v(z)=\left(5.10^{6}\right) \exp \left(-0.15\left(z-h^{\prime}\right)\right) \quad / \mathrm{s}$

where $h^{\prime}=70 \mathrm{~km}$ and $z$ is in $\mathrm{km}$. The ground is modelled as a conducting half space. Earth curvature is currently not considered. Details of the code and of the theory will be found in Baba and Hayakawa $(1995,1996)$. The upper height of the simulation box is $100 \mathrm{~km}$, well above the reflection height of $85 \mathrm{~km}$. At the upper boundary the tangential field components are matched to those of an upgoing wave in a semi-infinite half space above $z=100$ $\mathrm{km}$. At the downstream end of the box the boundary condition is one which can be obtained from the modal function expansions and is known as the finite element boundary integral method as proposed in McDonald and Wexler (1972). The code analyses the field in terms of the full set of TM modes in the waveguide. The incident field is assumed to consist of one TM mode only, either the dominant mode (No. 2) or the semi-Earth detached mode (No. 1). The principal output of the code consists of 2D plots in $x$ and $z$ of Trimpi amplitude and phase perturbations. In this paper we shall only calculate Trimpis on the ground downstream from the LIE. The scattered field at the downstream end of the box will be expanded in TM modes, thus giving the modal scattering matrix $M_{i j}$ for the strip perturbation. This may be defined as the complex amplitude of normalised mode $j$ at the downstream end of the box when the incident field is pure mode $i$ of unit amplitude.

\subsection{The Born $3 D$ code}

Full details of this code will be found in Nunn (1997). The ionospheric profiles $N e, v$ are exactly as above for comparison purposes. The ambient field $B o$ has magnitude and direction appertaining to the mid-point of the path, and the ground is assumed to have the conductivity and the dielectric constant of sea water, although some of the path is over land. This code does not allow horizontal inhomogeneity of the background ionosphere, although the version of Yeo (Clilverd et al., 1998) does. NWC and the receiver at Dunedin are assumed to be vertical electric dipoles. The LIE $\delta N e(x, y, x)$ is modelled as a Gaussian in $x$ and $z$ exactly as above, and the $y$ dependence is also Gaussian with a scale length $S y=2.75 S_{x}$, i.e., the LIE is elliptically shaped with the long axis perpendicular to the direction of propagation, and with ellipticity $e=S_{y} / S_{x}=2.75$. In the case $S_{x}=10 \mathrm{~km}$, an ellipticity of 10 was used in view of the small size of the LIE. Numerical experimentation has shown that the calculated Trimpis have only a very small dependence on the degree of ellipticity $e$, provided that this exceeds two. In this case the elongated elliptical patch behaves similarly to the strip perturbation used by the FEM code. The normal version of the code considers 12 modes (TE and TM) throughout, and computes Trimpis observed at Dunedin as a function of one or more LIE positional coordinates. For the purpose of comparing with the FEM code only,we may restrict the incident zero order field to consist of one TM mode only and compute Trimpis at the 


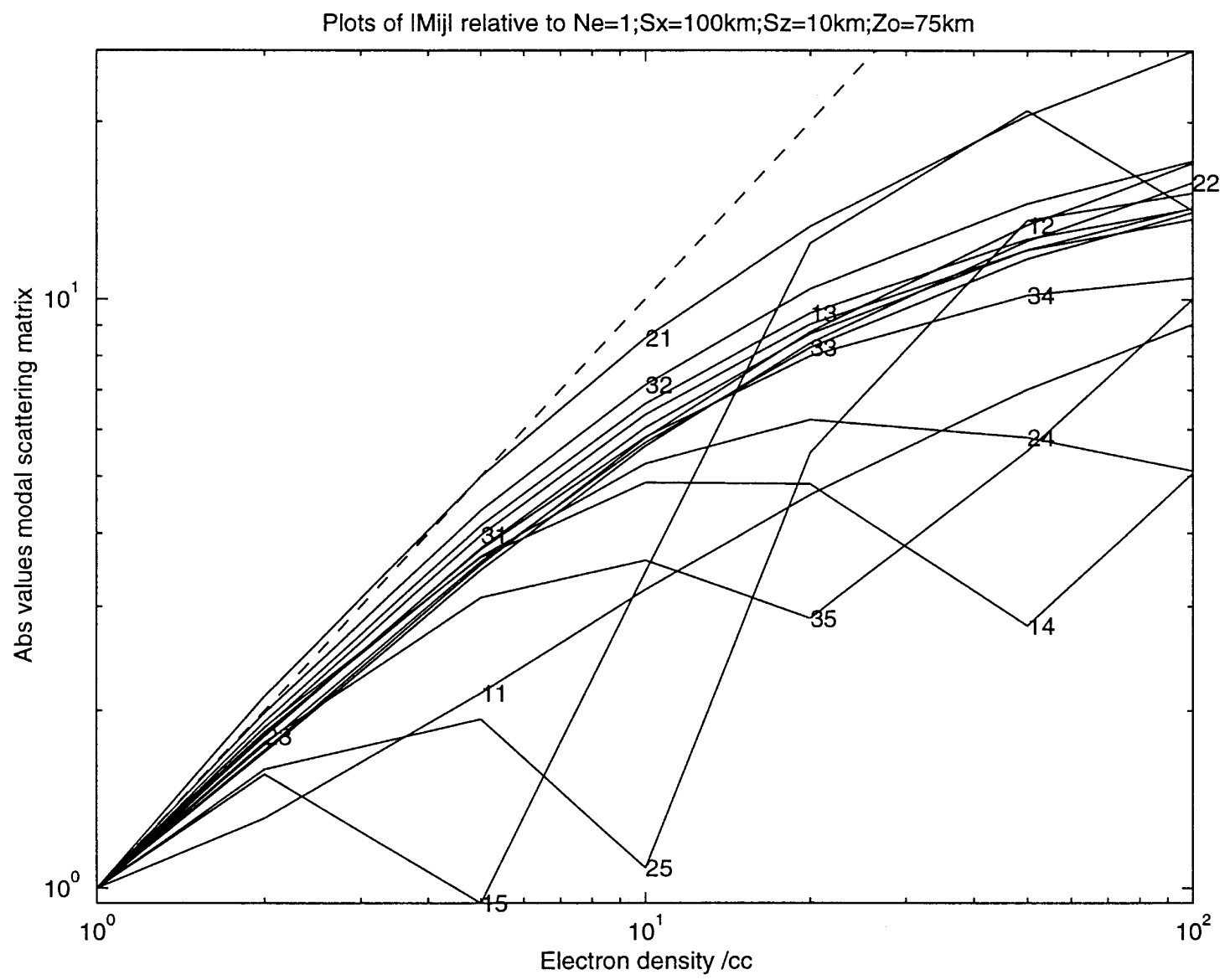

Fig. 1. FEM 2D code. Plot of modal scattering matrix elements $\left|M_{i j}\right|$ as functions of electron density perturbation $\delta N e_{0}$. The strip perturbation LIE is assumed large with $S_{x}=100 \mathrm{~km}$. Each curve is normalised to unity at $\delta N e_{0}=1$. The dashed line represents linear 'Born' behaviour. Significant departure from Born behaviour occurs for $\delta N e_{0}>6$ electrons/cc.

ground as a function of distance from the LIE along the great circle path. To make the geometry as close as possible to that of the FEM code, ideally the distance NWC-LIE should be infinite. In practice we shall employ a value of $5.0 \mathrm{Mm}$ as for a LIE some $700 \mathrm{~km}$ from Dunedin. The Trimpis produced are indistinguishable from those produced by a transmitter at infinity. The code also computes the modal scattering matrix $M_{i j}$ for a column of ionisation at the LIE centre (see Nunn, 1997), which will be very close to the scattering matrix for the patch as a whole.

\section{Results}

We shall first investigate the behaviour of the modal scattering matrix $M_{i j}$ in the case of the FEM code. We consider a large patch $S_{x}=100 \mathrm{~km}$, and use $S_{z}=10 \mathrm{~km}$ and $Z_{0}=75 \mathrm{~km}$. In Fig. 1 we plot the magnitudes $\left|M_{i j}\right|$ as functions of maximum electron density perturbation $\delta N e_{0}$, over a range from 1-100 electrons/cc. Note that $\left|M_{i j}\right|$ corresponds to scattering from mode $i$ to mode $j$. Each curve is separately normalised to have a value of 1 at $\delta N e_{0}=1$ electron/cc. We consider the leading $6 \mathrm{TM}$ modes, Nos, 1-6, which correspond to Nos, 2, 3, 5, 8, 10, 12 as returned by MODEFNDR. We plot the 15 matrix elements corresponding to scattering from TM modes 13 to TM modes $1-5$. If the Born approximation holds then we expect the $\left|M_{i j}\right|$ to be proportional to $\delta N e_{0}$, which dependence is shown on the graph as a dashed line. Departure from this line becomes marked at about 6 electrons/cc and indicates non-Born behaviour, and progressive exclusion of the incident field $E_{0}$ from the patch due to the skin depth effect. We may ignore $\left|M_{11}\right|$, which has a small value, since in the absence of Earth curvature mode 1 has little penetration at the main scattering level $\sim 75 \mathrm{~km}$. We note that the approach to linearity is fairly asymptotic, and rigorously Born behaviour is only 


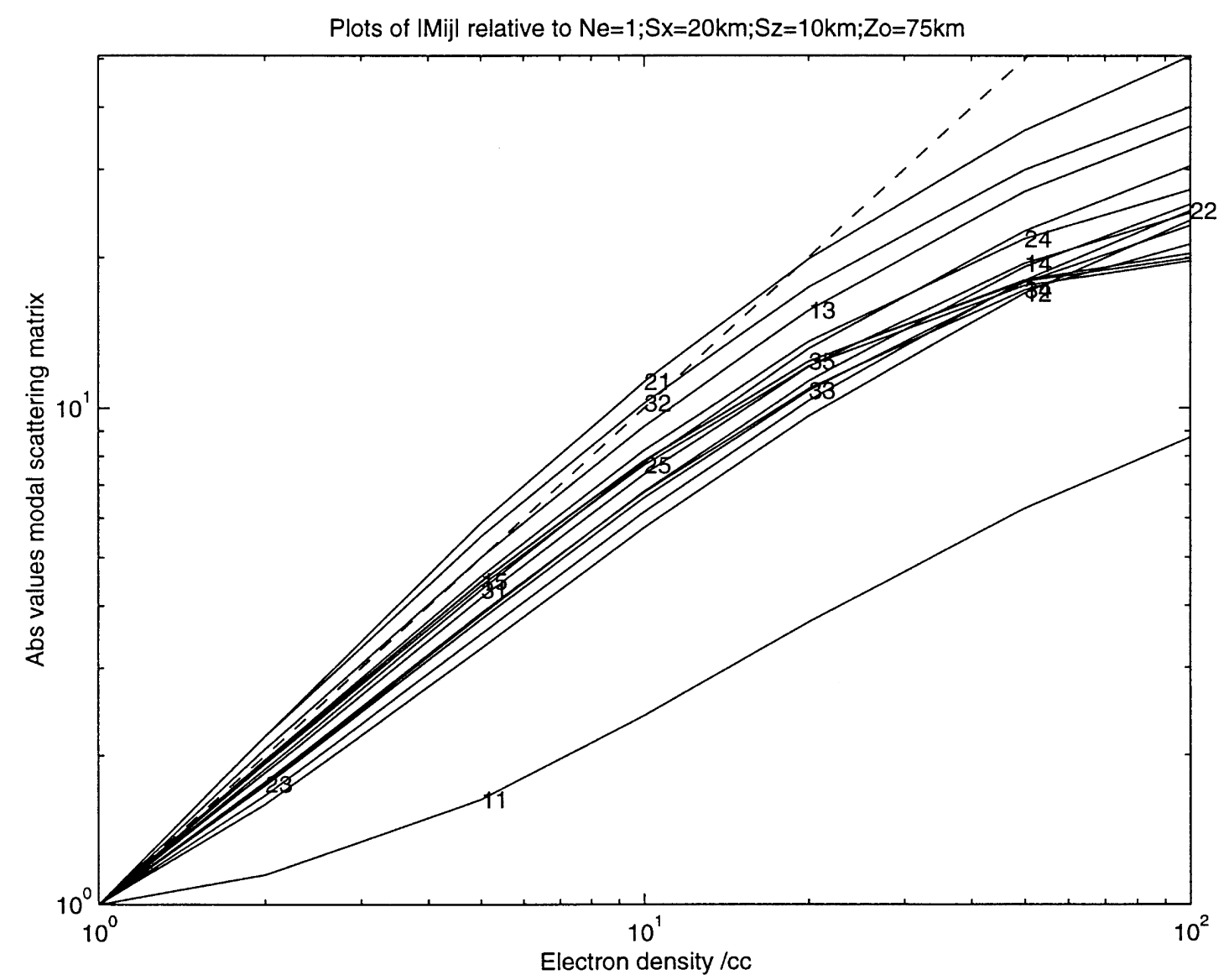

Fig. 2. FEM 2D code. Plot of absolute magnitudes of the dominant components of the modal scattering matrix $\left|M_{i j}\right|$ as functions of $\delta N e_{0}$ as in Fig. 1. A smaller strip perturbation with $S_{x}=20 \mathrm{~km}$ is used. Departure from Born behaviour now occurs for $\delta N e_{0}>20$ electrons/cc.

achieved at very small $\delta N e_{0}$. Similar results to the above were reported on by Baba et al. (1998), but only the three dominant TM modes at $S_{x}=100 \mathrm{~km}$ were investigated.

Figure 2 shows a similar plot but for a thinner strip LIE with $S_{x}=20 \mathrm{~km}$. Not surprisingly it is seen that the $\left|M_{i j}\right|$ closely follow a linear Born dependence on $\delta N e_{0}$ up to a value of about 20 electrons/cc. For some reason the elements 15, 25, 35, 14, 24 show markedly non Born behaviour for $S_{x}=100 \mathrm{~km}$ but not for $S_{x}=20 \mathrm{~km}$. Some elements of the matrix, notably $\left|M_{21}\right|$ and $\left|M_{32}\right|$, achieve values a little above the 'linear Born' line, particularly for the smaller path with $S_{x}=20 \mathrm{~km}$. This may be understood as being due to the relative phasing of the scattered mode when integrated across the LIE.

We thus see that the limit of the Born approximation is mainly determined by $\delta N e$, since the subionospheric waveguide field penetrates the patch from below. There is also a dependence on horizontal scale, however, with large patches being more non-Born than smaller ones. As a rule of thumb for horizontal scale sizes of 100 $\mathrm{km}$ the Born approximation will be valid for maximum electron density perturbations up to about 6 electrons/cc. For smaller scale sizes $\sim 20 \mathrm{~km}$, the Born approximation will hold up to values of about 20 electrons/cc. At both these upper limits, however, the 3D Born code will still overestimate the scattered field strength by some $20 \%$.

It will be of considerable interest to study the structure of the modal scattering matrix and particularly the extent to which it is non diagonal. Since the Born 3D code embodies Earth curvature and an anisotropic ionosphere, we shall use this code to analyse $\left|M_{i j}\right|$. Figure 3 presents a MATLAB visualisation of the modal scattering matrix $\left|M_{i j}\right|$, which is here defined as the scatter matrix of a column of perturbed ionisation at the patch centre, arbitrarily normalised to $\left|M_{22}\right|(\mathrm{Nunn}, 1997)$. Parameters used are $S_{x}=100 \mathrm{~km}, S_{z}=10 \mathrm{~km}, Z_{0}=75 \mathrm{~km}$, and $\delta N e=10$ electrons/cc. We first present the submatrix defined by the 6 first TM modes at this frequency. These are numbered 1-6, and shadow MODEFNDR's modes 2, 3, 5, 8, 10,12 . We note first the substantial off-diagonal elements 


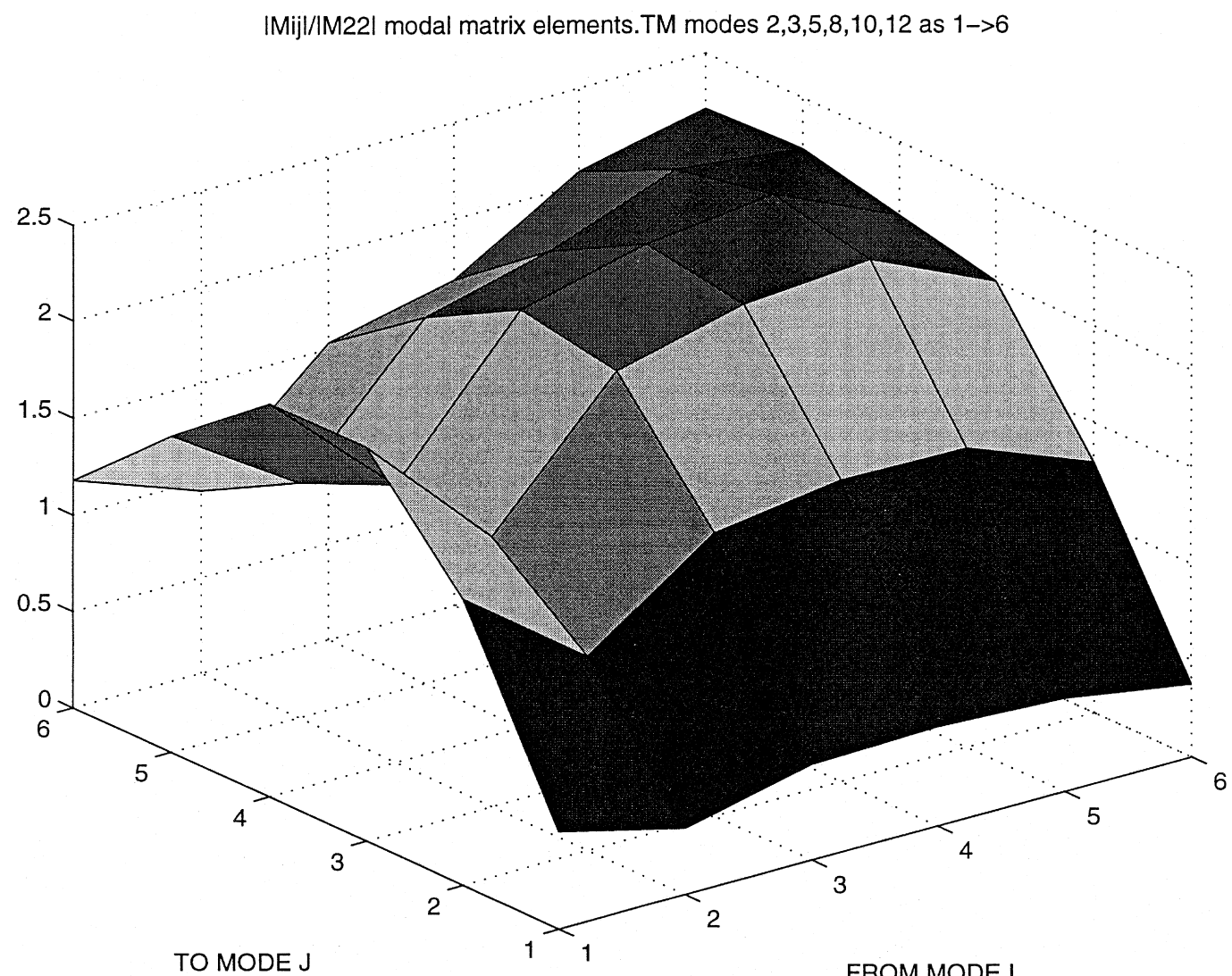

Fig. 3. 3D Born code. Matlab surface visualisation of the magnitude scattering matrix $\left|M_{i j}\right|$ for the first six dominant TM modes only. The scattering is from mode $i$ to mode $j$. Note the substantial cross coupling between modes.

over the whole surface and particularly the strong coupling from mode 1 (the semi-Earth detached mode with low excitation factor) to dominant TM modes 2 and 3. Figure 4 visualises the entire scatter matrix $\left|M_{i j}\right|$, viewed as a MATLAB shaded surface, and Fig. 5 presents the same data as a contour plot. The mode ordering is the 6 TM modes first (MODEFNDR Nos, 2, 3, 5, 8, 10, 12), followed by the 6 TE modes (MODEFNDR Nos, 1, 4, $6,7,9,11)$, the latter of course having little impact on the Trimpi result. The scattering between TE modes appears to be mainly diagonal, peaking for $M_{10,10}$. There is substantial coupling from TE modes to TM modes, peaking for $M_{10.4}$. The lowest order TE mode (here No. 7) scatters very strongly into modes 4 and 10 . Scattering from TM to Te modes appears weak, $\left|M_{6,10}\right|$ being the largest component.

These results for $\left|M_{i j}\right|$ may be readily understood from the modal expansion of scattered field presented in Nunn (1997). A 'point' scatterer located at a height $z$ will give a modal scattering matrix $N_{i j}$ given by

$N_{i j}=\Gamma_{i}(z) \Gamma_{j}(z) \sigma_{z z}^{\prime}(z) \lambda_{33}^{j}$ where $\Gamma_{i}(z)$ is the height gain functions for mode $i, \sigma_{z z}^{\prime}(z)$ is the $z z$ component of the perturbation of conductivity tension and $\lambda_{33}^{j}$ is the excitation factor of scattered mode $j$. If only mode $j \rightarrow$ mode $j$ scattering is considered, i.e., a unimodal problem, then a useful expression for scatter phase $\psi$ at the receiver may be derived. It is

$$
\begin{aligned}
\psi=\angle E_{s}- & \angle E_{0}=225^{\circ}+2 \angle \Gamma_{j}(z)+ \\
& \angle \sigma_{z z}^{\prime}(z)+\angle \lambda_{33}^{j}-(P D) 360^{\circ} / \lambda \text { degrees }
\end{aligned}
$$

where $(P D)$ is the path difference between the direct and scattered paths. The scatter matrix for a column $M_{\mathrm{ij}}$ will be simply the integral over $z$ of the above, i.e.,

$M_{i j}=\int N_{i j} \mathrm{~d} z=\int \Gamma_{i}(z) \Gamma_{j}(z) \sigma_{z z}^{\prime}(z) \lambda_{33}^{i} \mathrm{~d} z$

We see at once that $M_{i j}$ and $M_{j i}$ will have the same phase and will have values that differ only by the ratio of the complex excitation factors $\lambda_{i}$ and $\lambda_{j}$. In fact $\left|M_{i j}\right|$ is approximately determined by the penetration of modes $i$ and $j$ at the height where $\sigma_{z z}^{\prime}$ is maximum, which is about $75 \mathrm{~km}$. 


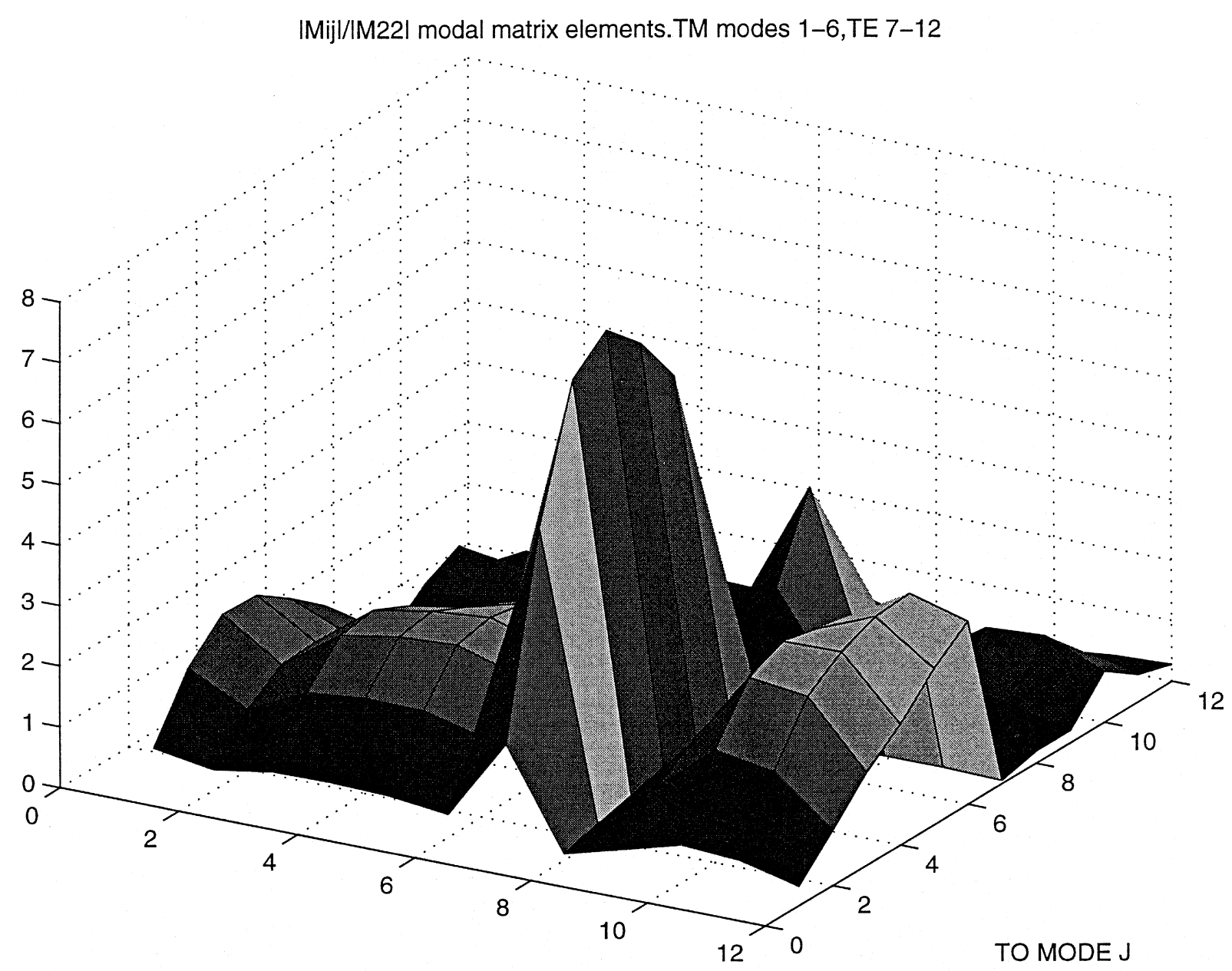

FROM MODE I

Fig. 4. 3D Born code. Magnitude scattering matrix $\left|M_{i j}\right|$ for 12 modes, ordered TM 1-6, TE 7-12, as a MATLAB surface visualisation.

It is a matter of considerable interest to compare the modal scattering matrices returned by the FEM 2D code with those of the 3D Born code. Baba et al. (1998) made such a comparison for the case where the Born 3D code was run with an isotropic ionosphere, and found very good agreement. In Fig. 6 we compare the $\left|M_{i j}\right|$ matrix from the FEM code with that calculated in the 3D Born code with an anisotropic ionosphere. We take the case $\delta N e_{0}=10$ electrons $/ \mathrm{cc}, z_{0}=75 \mathrm{~km}, S_{z}=10 \mathrm{~km}$. In a 3D geometry, the matrix $\left|M_{i j}\right|$ does not have the same obvious meaning as in the 2D case, so we compare matrices normalised to $\left|M_{22}\right|$ for $S_{x}=20$ and $100 \mathrm{~km}$. Looking at the top panel we see that the matrix structures are very similar, with the exception of $\left|M_{12}\right|$ and $\left|M_{13}\right|$, which the FEM code underestimates due to the absence of Earth curvature in the model. Also $\left|M_{23}\right|$ is somewhat larger in the case of the FEM code. The bottom panel also exhibits a similar correlation, even though this case is more nonBorn like. The obvious features are: (a) diagonal components $\left|M_{j i}\right|$ increase with mode order $j$.

(b) strong two way coupling between the dominant TM mode $(j=2)$ and the next TM mode $(j=3)$.

(c) weak coupling from modes 2, 3 to the Earth detached $\operatorname{mode}(j=1)$.

(d) strong coupling from the Earth detached mode $(j=1)$ to TM modes $(j=2,3)$, small without Earth curvature.

The occurrence of strong intermodal scattering was shown theoretically by Wait $(1991,1995)$ and our results are in full accord with his findings.

We will now compare amplitude and phase Trimpis on the ground, computed by each code as a function of $x^{\prime}$, the Great Circle Path distance downstream from the LIE centre. In Baba et al. (1998) such a comparison was made for the case where the incident field consisted of the dominant TM mode No. 2 only. With the Born 3D code 
IMij//IM22| modal matrix elements.TM modes 1-6,TE 7-12

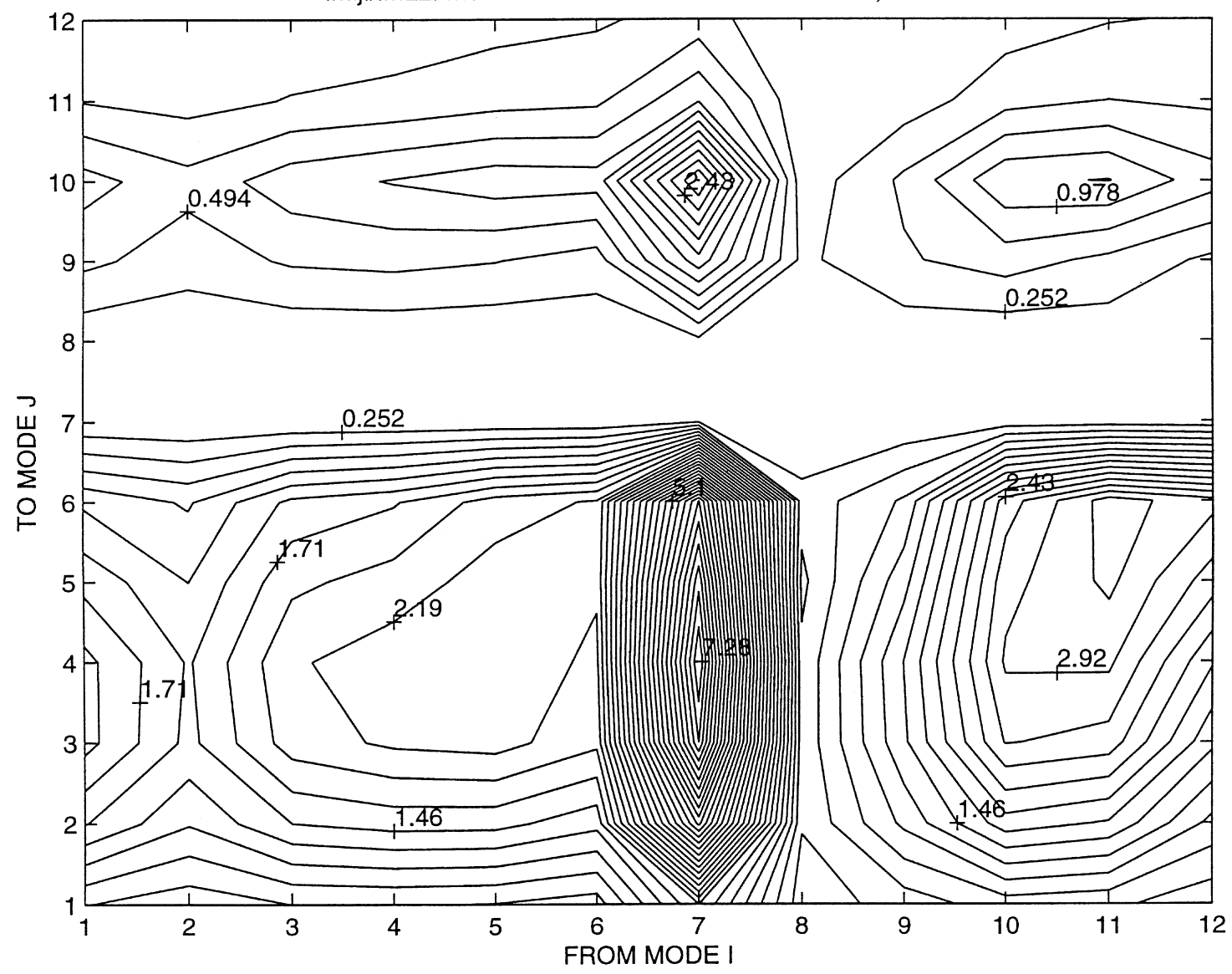

Fig. 5. 3D Born code. Magnitude scattering matrix $\left|M_{i j}\right|$ for all modes, ordered TM 1-6, TE 7-12, as a contour plot.

run with an isotropic ionosphere, excellent agreement was obtained. We wish to extend these comparisons to the case where the Born 3D code is anisotropic and also for incident TM mode 1. The top panels of Figs 7 and 8 plot this result for the $3 \mathrm{D}$ Born code with anisotropic ionosphere, using $\delta N e=10$ electrons $/ \mathrm{cc}, z_{0}=75 \mathrm{~km}$, $S_{z}=10 \mathrm{~km}$, and for 3 values of $S_{x}=10,50,100 \mathrm{~km}$. Note that the large patch with $S_{x}=100 \mathrm{~km}$ would appear to be outside the Born regime, but it is desirable that the comparison span both Born and non-Born regimes. The transmitter 'NWC' at $f=22.3 \mathrm{kHz}$ is placed $5 \mathrm{Mm}$ from the patch, and the code is modified such that only the dominant TM mode No. 2 (MODEFNDR No. 3) is incident on the LIE. The patch has an ellipticity of 2.75 and is placed at right angles to the $x$ axis. The bottom panels of Figs 7 and 8 are the corresponding plots for the FEM code using the same data. Inspecting Fig. 7 we see that the amplitude Trimpis are highly similar, with a double negative peaks at $x=550 \mathrm{~km}$, a negative peak at $x=2600 \mathrm{~km}$ and a small negative peak at $x=1600 \mathrm{~km}$. Furthermore the amplitudes of the predicted Trimpis are almost the same. The Born code has more 'oscillations', but when run in isotropic mode (Baba et al., 1998), these disappear and the agreement is even better. Interestingly, the ratio of the Trimpis for the three horizontal scales $S_{x}$ is about the same in both cases.

The phase Trimpi plots (Fig. 8) show a similar close resemblance. Large positive peaks $\sim 12$ degrees will be seen at 400, 1400 and $3000 \mathrm{~km}$. Again the relative size of the phase Trimpis for the three values of $S_{x}$ is similar in both cases, and the 3D Born results are more oscillatory. The agreement shown in Figs 7 and 8 is surprisingly good, considering the fact that the two codes are based upon totally different computational approaches. Presumably an elongated ellipse perpendicular to the $x$ axis behaves very much as a strip perturbation, since most scattering is in the forward direction. The main differences between the codes are then Earth curvature and ionospheric anisotropy, neither of which appear to compromise this comparison.

Figure 9 shows the magnitude Trimpis downstream from the LIE for both the Born 3D code with anisotropic ionosphere and the 2D FEM code. The calculations are the same as in Fig. 7 except that the incident field is 
Comparison of modal scattering matrices $\mid \mathrm{Mij} / / \mathrm{M} 22 \mathrm{I}$;Sx=20kms
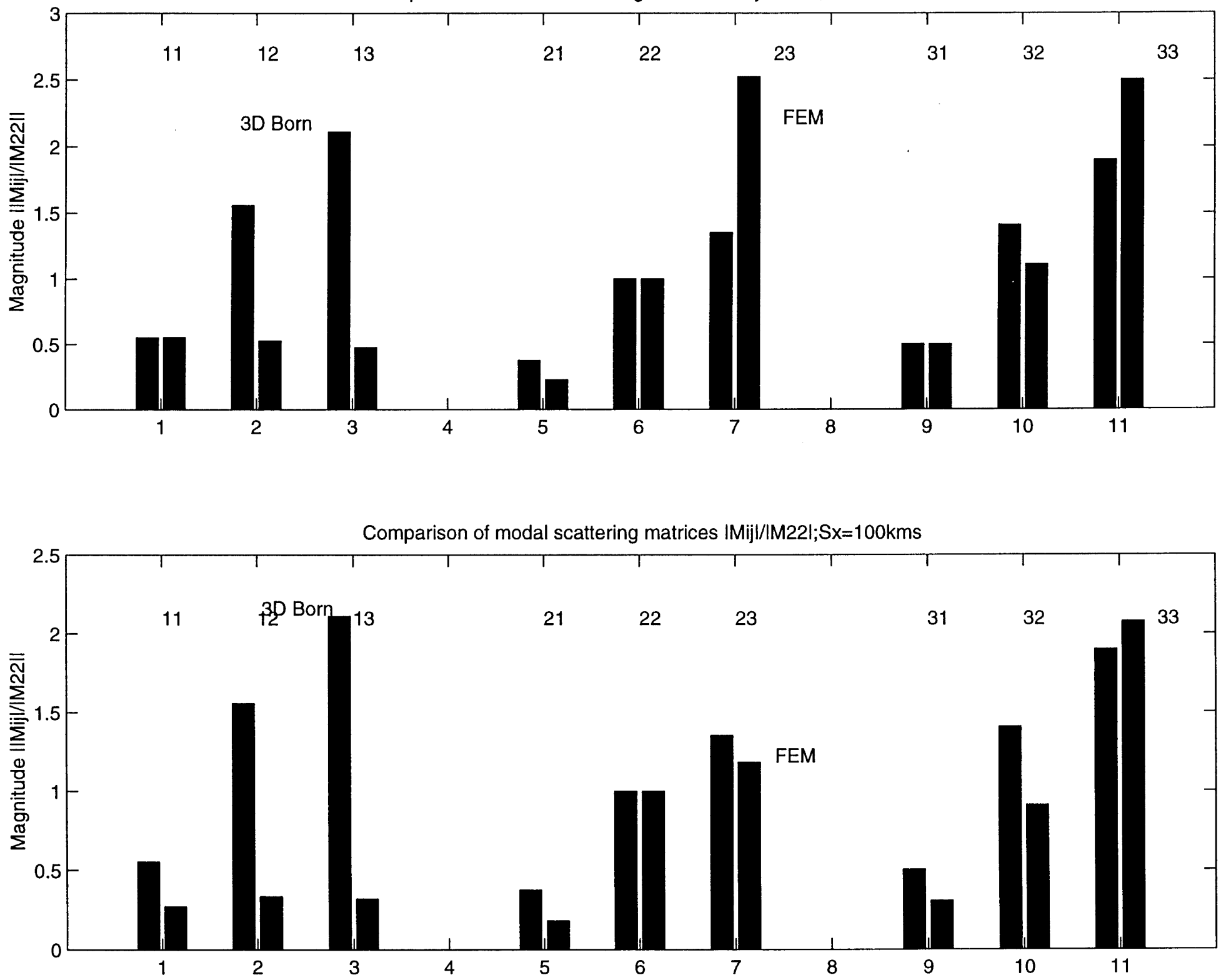

Fig. 6. Comparison of the structure of the modal scattering matrices $\left|M_{i j}\right|$ for the FEM and 3D Born code with anisotropic ionosphere. Elements are normalised to $\left|M_{22}\right|$ for comparison purposes. The close resemblance is obvious. 

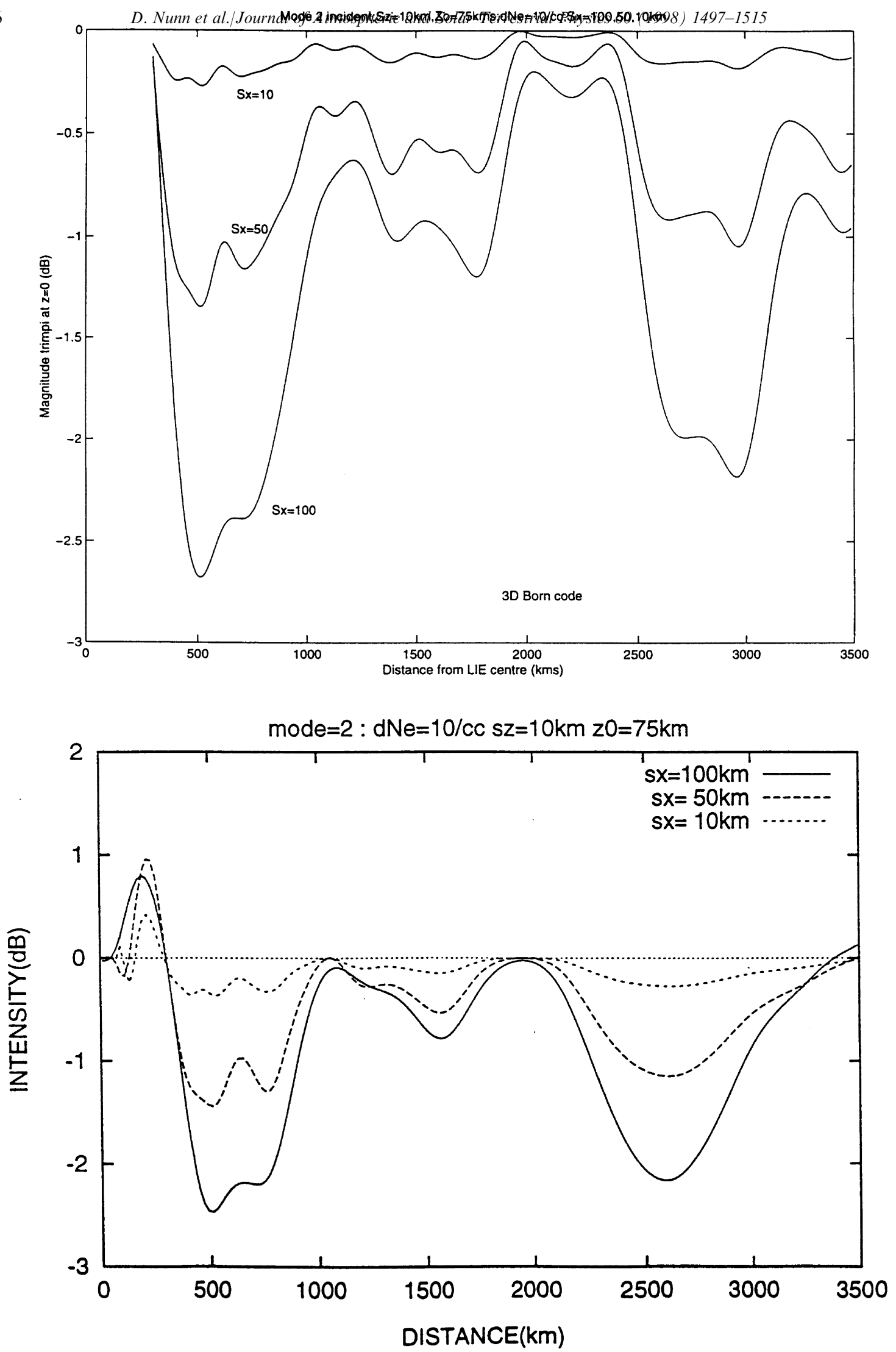

Fig. 7. Upper panel: 3D Born code with anisotropic ionosphere. Ground magnitude Trimpis as a function of Great Circle Path distance downstream from the LIE centre. Curves are for three values of $S_{x}=10,50,100 \mathrm{~km}$. Only the dominant TM mode 2 is assumed incident. Lower panel: same calculation with 2D FEM code. The very close similarity will be noticed. 


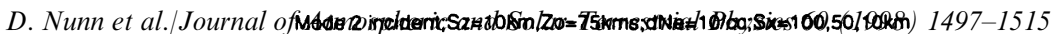
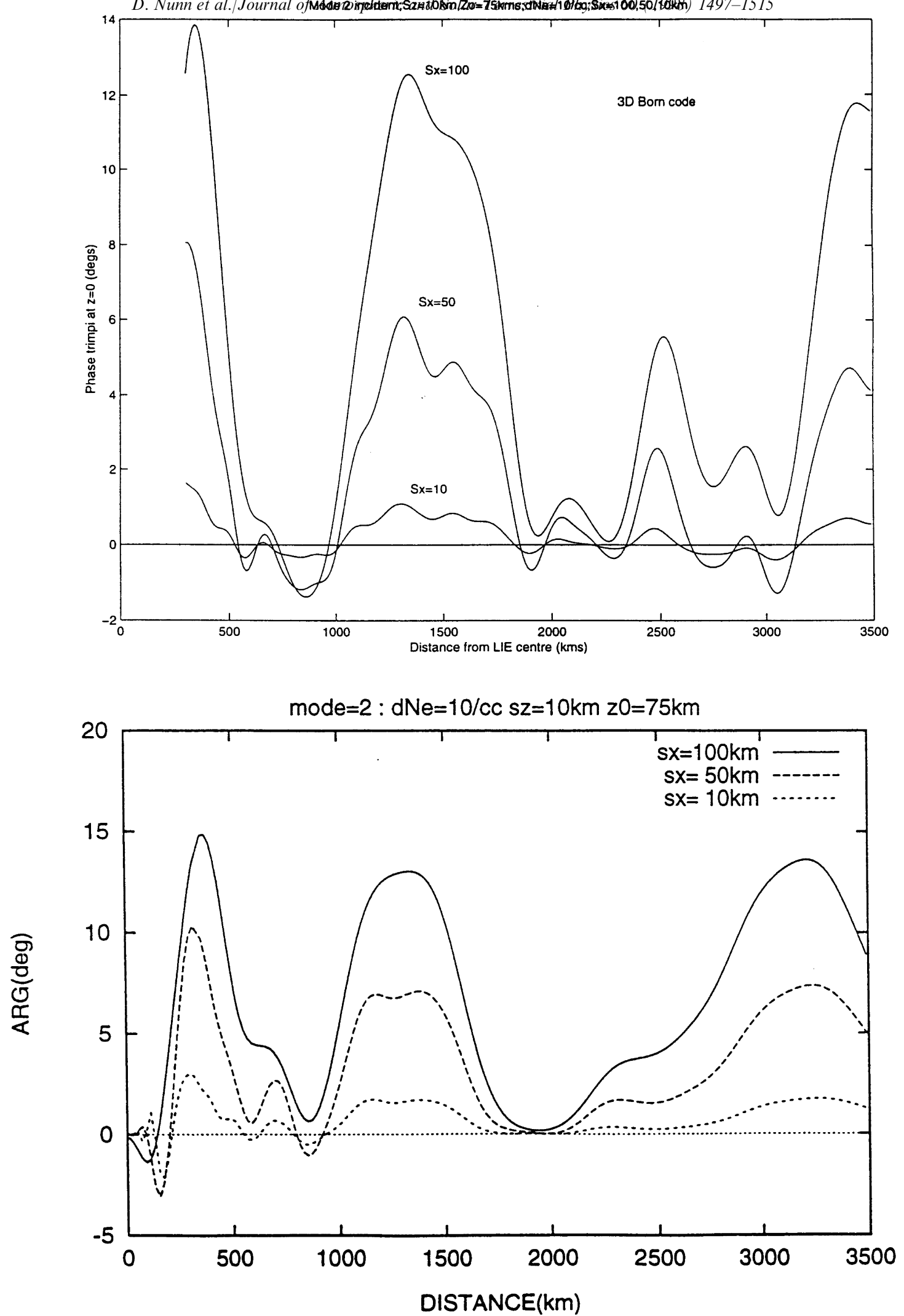

Fig. 8. Upper panel: 3D Born code with anisotropic ionosphere. Ground phase Trimpis as a function of Great Circle Path distance downstream from the LIE centre. Curves are for three values of $S_{x}=10,50,100 \mathrm{~km}$. Only the dominant TM mode 2 is assumed incident. Lower Panel: same calculation with the 2D FEM code. The similarity is obvious. 

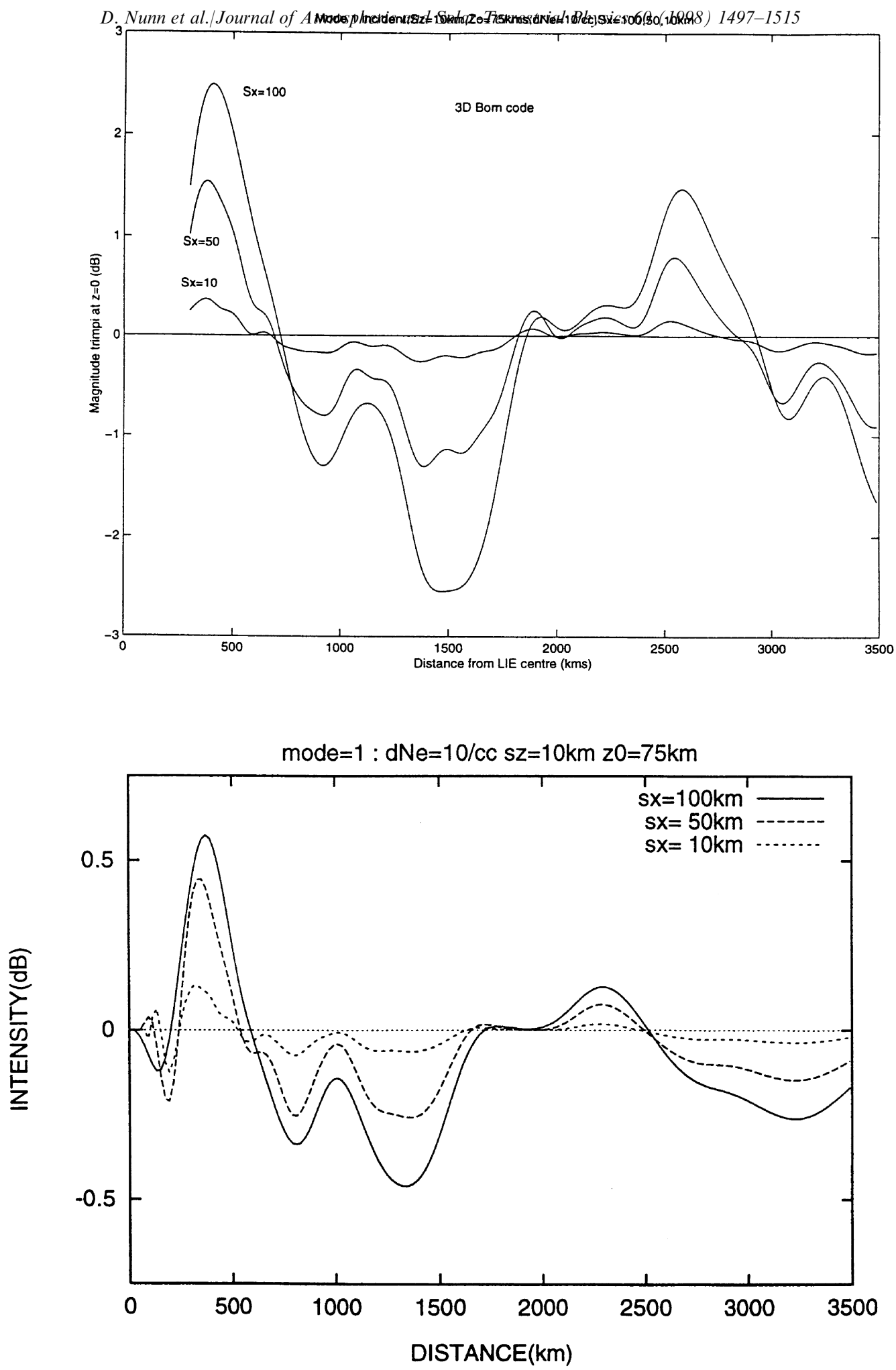

Fig. 9. Upper panel: 3D Born code with anisotropic ionosphere. Ground magnitude Trimpis as a function of Great Circle Path distance downstream from the LIE centre. Curves are for three values of $S_{x}=10,50,100 \mathrm{~km}$. Only the TM mode 1 is assumed incident.Lower panel: same computation with 2D FEM code. The functional similarity is very close, but the results for the 3D Born code are overall about four times larger due to the low penetration of mode 1 into the ionosphere in the case of the FEM code which does not model Earth curvature. 
assumed to consist purely of TM mode 1, which MODEFNDR labels the Earth detached mode No. 2 Fig. 10 plots the corresponding phase Trimpis. Clearly the results from the two codes have a very high correlation, but the overall Trimpi magnitudes are about four times as large in the Born 3D case. The reason for this is at once apparent. Mode 1 has a very weak penetration into the $\mathrm{D}$ region in the 2D FEM code due to the absence of Earth curvature.

The two codes make rather different assumptions regarding scattering geometry, Earth curvature and ionosphere anisotropy, and use a totally different theory and computational methods. The extremely close correspondence between the two sets of results proves considerable verification of both codes and their underlying theory.

We finally present computations of Trimpis at Dunedin from NWC at the new frequency of $f=19.8 \mathrm{kHz}$, using the 3D Born code. All 11 modes returned by MODFNDR will be used throughout. Parameters are set at realistic levels but within the Born limits. We take $\delta N e_{0}=5$ electrons/cc and again assume a 3D Gaussian distribution with $S_{x}=70 \mathrm{~km}, S_{y}=140 \mathrm{~km}$ and $S_{z}=10$ $\mathrm{km}$. Average patch height is $z_{0}=75 \mathrm{~km}$, and the elliptical patch is oriented E-W. Figure 11 shows zero order, perturbed and total electron densities as a function of height at the patch centre. Figure 12 plots magnitude Trimpis at Dunedin as a function of $x$, the distance from NWCLIE, as the patch is moved along the Great Circle Path. Trimpis up to $\sim-1 \mathrm{~dB}$ are predicted, with some variability due to modal interference. Figure 13 shows the phase Trimpis, whose values range from $+0 \rightarrow 6$ degrees. Figure 14 shows scatter phase as a function of $x$. It is seen to be nearly always in the $0-180$ degree quadrant, and usually in the 90-180 degree quadrant, giving classical $(-+)$ Trimpis, but it oscillates considerably due to multimodal effects. Figure 15 plots scatter magnitude, relative to the direct field, as a function of $x$. Interestingly there is a very pronounced maximum mid path, as well as at both ends near the transmitter or receiver. The mid point peak would appear to have the following explanation. Trimpis are expected to have an underlying amplitude variation as (R1.R2) $)^{-1 / 2}$ along the great circle path, which is a fairly weak dependence except close to the transmitter or receiver. At the midpoint signals travelling rom NWC-Dunedin via mode 2 /mode 3 will be cophasal with mode $3 /$ mode 2 signals, and so on. This effect may be relevant to Seismo Trimpis (O.A. Molchanov, private communication), which are large weak D region inhomogeneities allegedly produced by effects in the Earth's crust immediately prior to an earthquake. (Molchanov et al., 1998). It will also be noticed that Trimpis produced by this patch, which is not far off non-Born like, are of average size. Larger Trimpis, often observed, must clearly come from inhomogeneities that are either much stronger/larger or much closer to either transmitter or receiver. Such patches will undoubtedly be non-Born like and require FEM codes for really accurate modelling.

\section{Conclusion}

This paper has set out to investigate the scattering of subionospheric VLF radiation by night-time D region ionospheric inhomogeneities or LIEs. This work confines itself to the so-called classical Trimpis where the patches of enhanced ionisation are produced by energetic particle precipitation due to lightning whistlers. The problem selected has been that of Great Circle Path Trimpis produced at Dunedin, NZ, on transmissions from NWC, Australia.

Two entirely separate modelling codes have been used to look at this problem. The first is a finite element method code (FEM) that is rigorous in the strong scattering limit (non-Born). Due to the complexity of the problem the code models the scattering process in two dimensions, and currently does not invoke ionospheric anisotropy or Earth curvature. The second model is a weak scattering (Born) model, which is fully 3D and includes Earth curvature and ionospheric anisotropy. Results from the two codes have been compared and, in view of the considerable differences in geometry, the results are surprisingly close. This tends to confirm the validity of both modelling techniques within the bounds of their appropriate parameter ranges. Considerable attention has been focussed on the modal scattering matrices in both cases. Again these have very similar structures, and both models point to strong intermodal scattering between the dominant TM modes. More detailed analysis of the scattering matrix from the FEM code has shown the extent of validity of the Born approximation. Roughly speaking a LIE of radius $100 \mathrm{~km}$ will become non-Born like at a maximum electron density perturbation of about 6 electrons/cc. Calculations of Trimpis at Dunedin using the 3D Born code and a patch strength not far off the Born limit produced Trimpis of average size only. The very strong Trimpis observed must be related to powerful patches, requiring non-Born techniques.

The path for future research on this problem is clear. For the FEM approach extension to 3D modelling, and inclusion of Earth curvature and ionospheric Bo field, are called for. Detailed modelling will require an appropriate mix of incident modes. Work on all these upgrades is well underway at present. Much recent research focuses on Trimpis produced by Sprites and by LIEs produced directly by lightning discharges. A 3D FEM code will be ideally suited to compute scattering from small intense structures, such as sprite columns.

The 3D Born code may be uprated by incorporation of an attenuation function for the incident zero order 

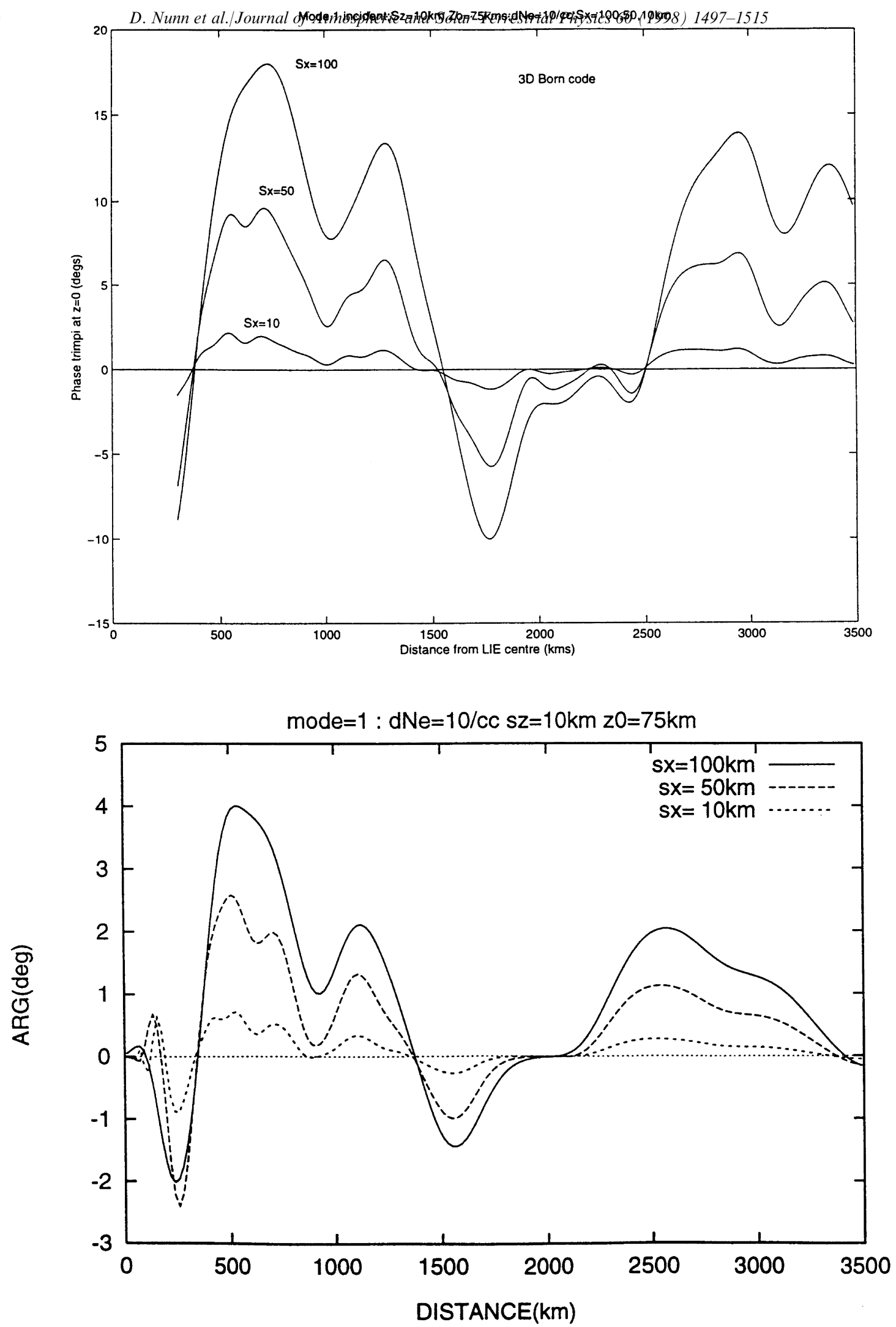

Fig. 10. Upper panel: 3D Born code with anisotropic ionosphere. Ground phase Trimpis as a function of distance downstream from the LIE centre, with only TM mode 1 incident. Curves are for three values of $S_{x}=10,50,100 \mathrm{~km}$. Lower panel: same computation with FEM code. The functional similarity is very close, but again with a factor $\sim 4$ difference. 


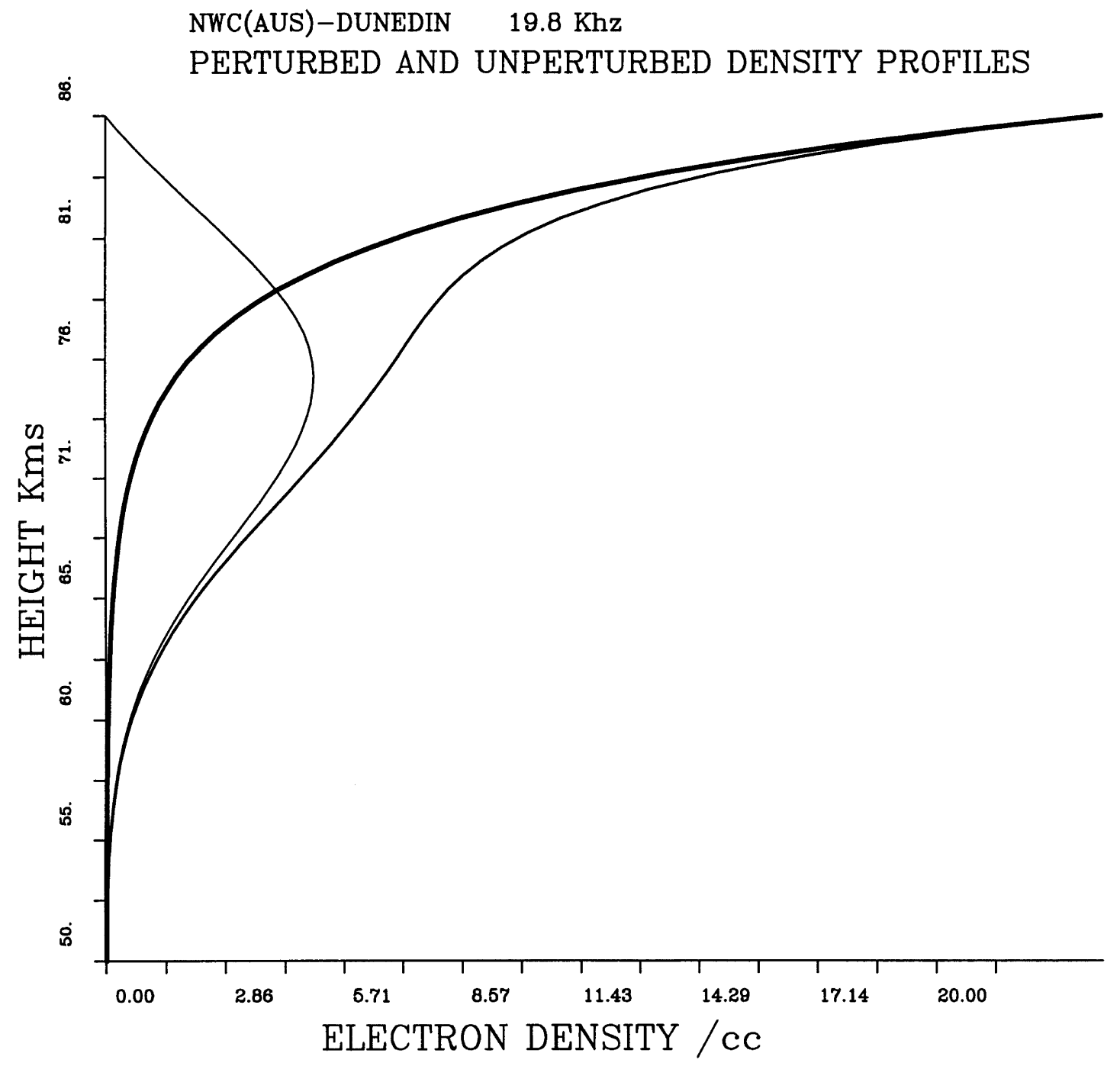

Fig. 11. Zero order, perturbed and total electron densities at the LIE centre, as a function of height, as used for modelling Trimpis on the path NWC-Dunedin. 


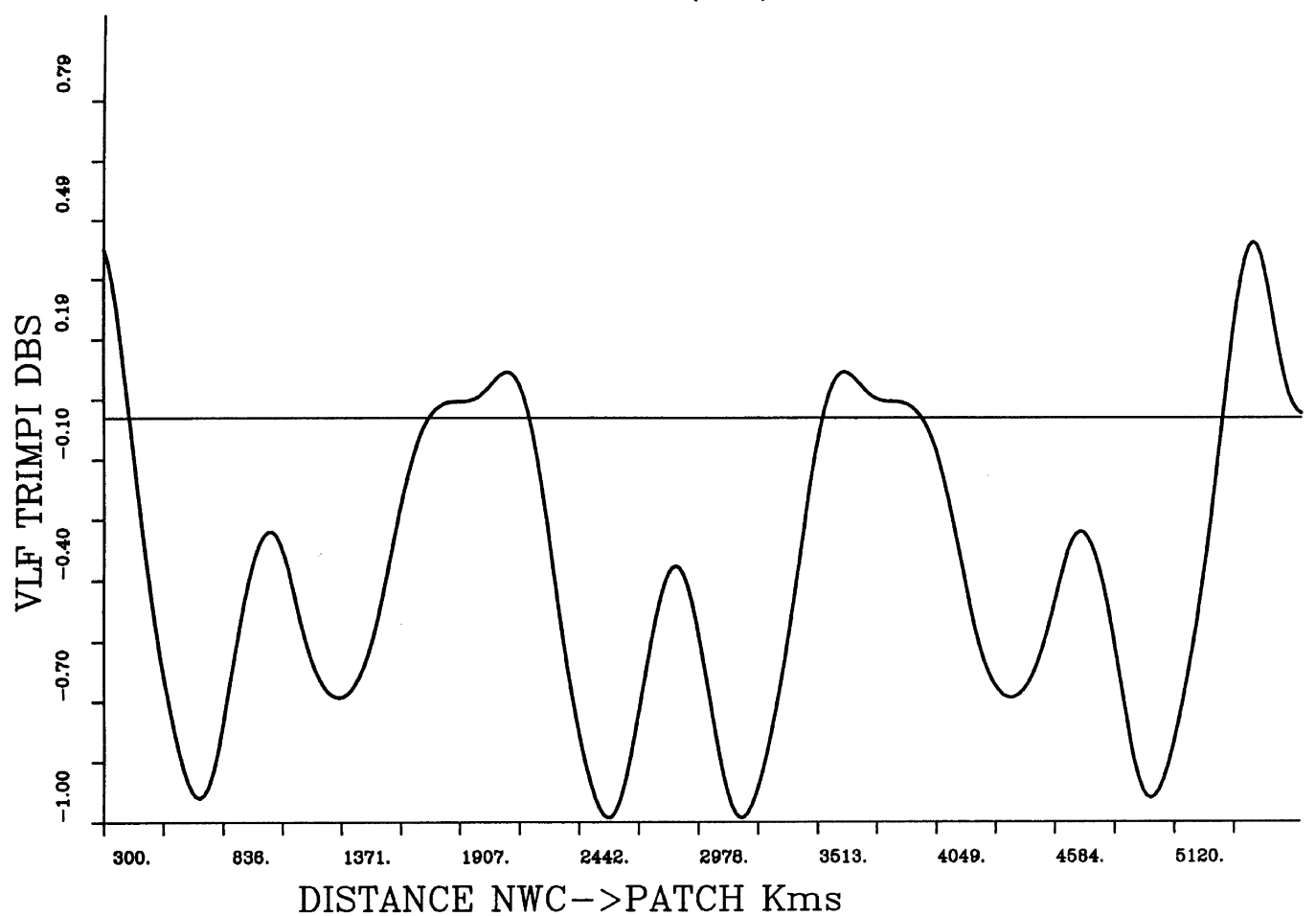

Fig. 12. 3D Born code with anisotropic ionosphere. Calculation of magnitude Trimpis observed at Dunedin as a function of Great Circle Path distance from NWC to the LIE.

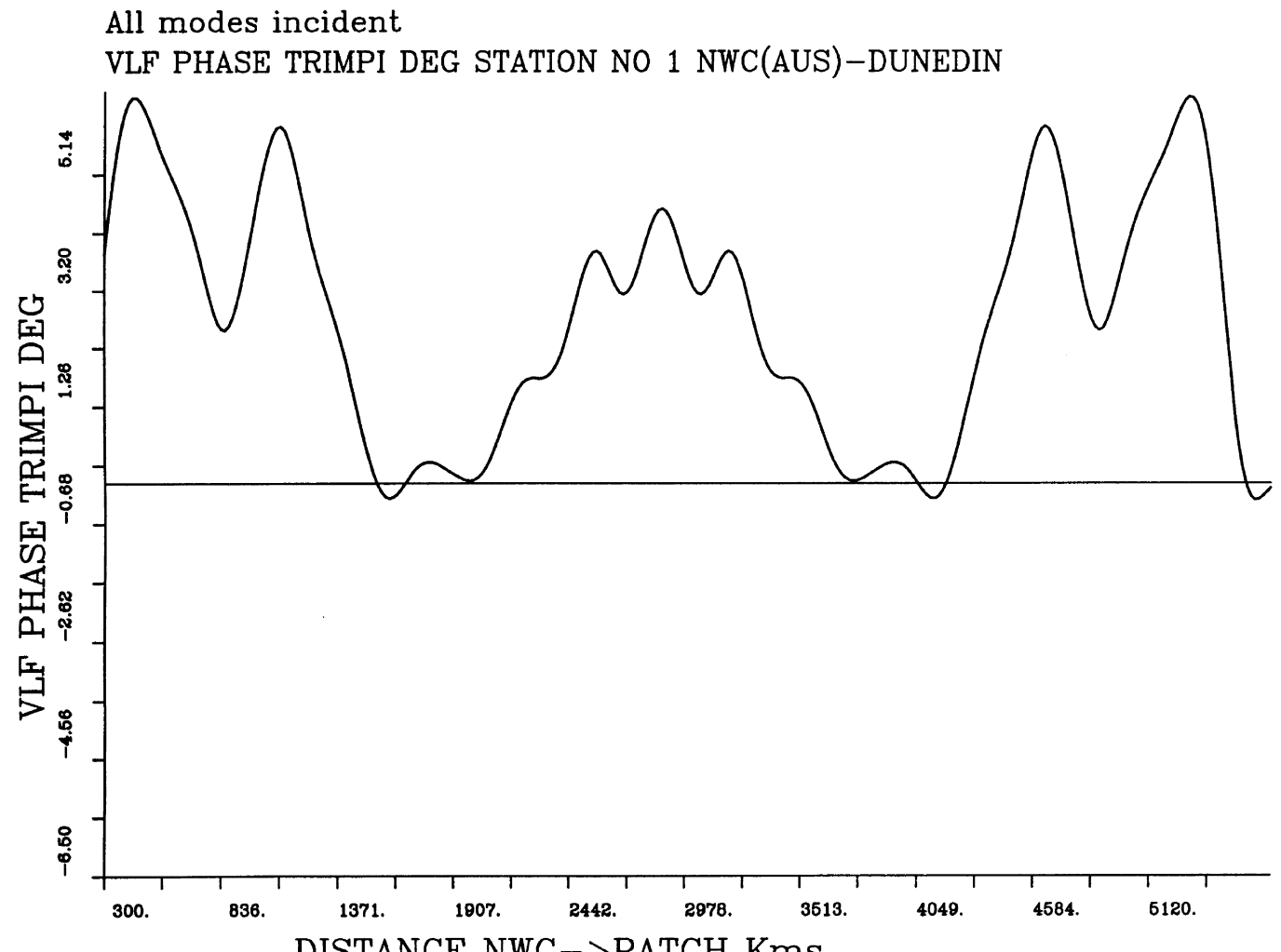

Fig. 13. 3D Born code with anisotropic ionosphere. Calculation of phase Trimpis observed at Dunedin as a function of Great Circle Path distance from NWC to the LIE. 
All mbdes nimeidental of Atmospheric and Solar-Terrestrial Physics 60 (1998) 1497-1515

SCATTER PHASE DEGS STATION NO 1 NWC(AUS)-DUNEDIN

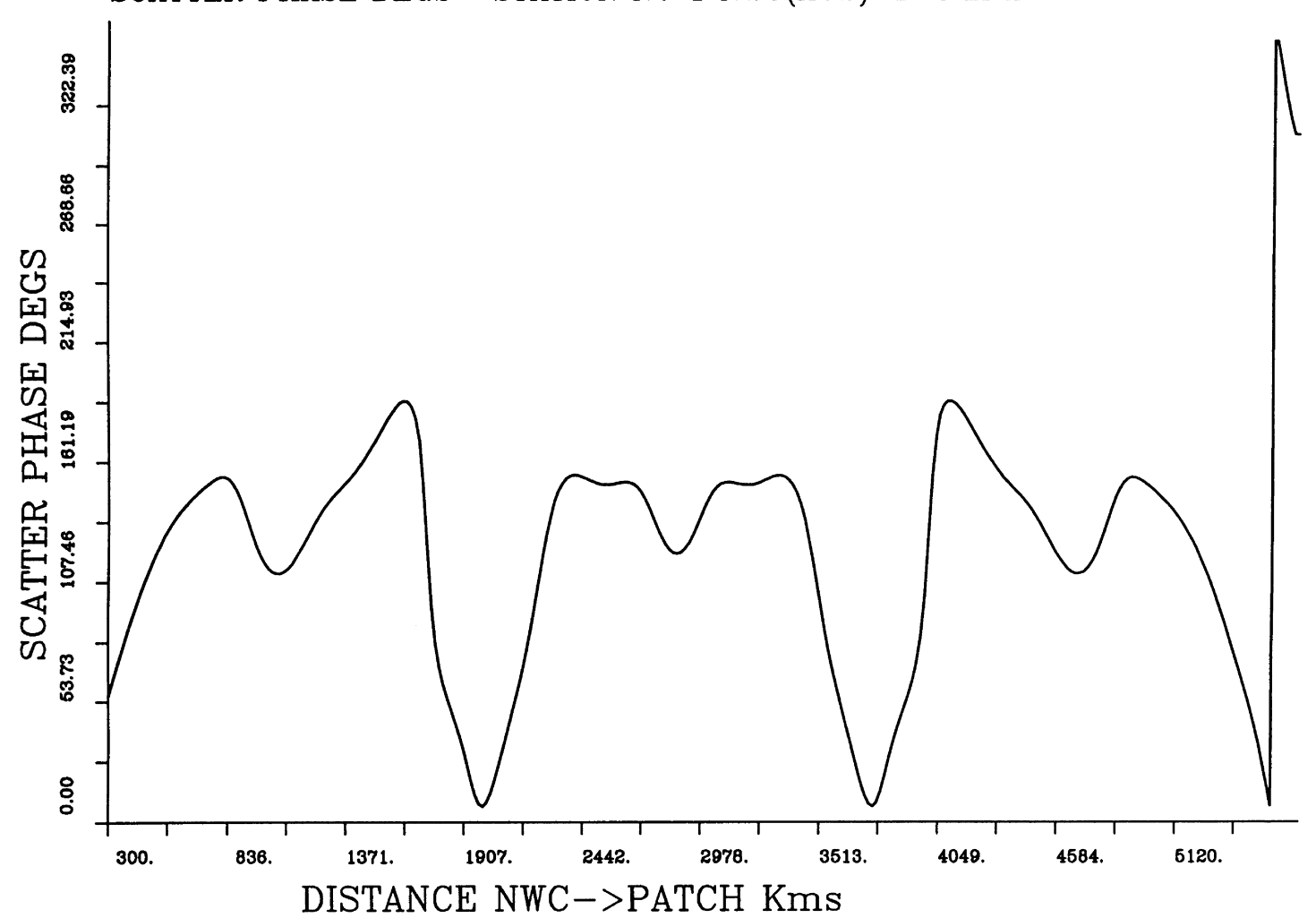

Fig. 14. Scatter phase as a function of Great Circle Path distance from NWC to the LIE.

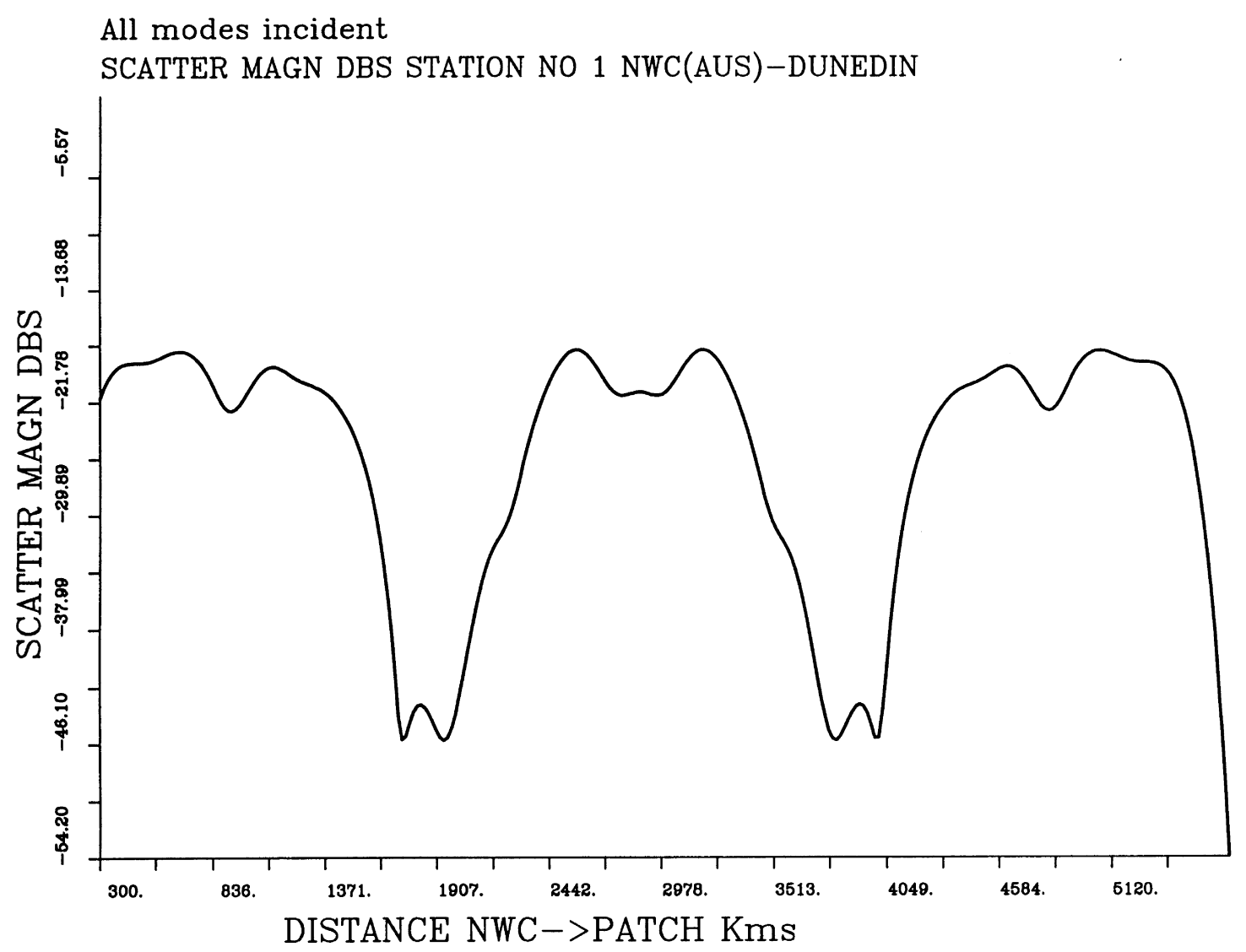

Fig. 15. Relative scatter field magnitude (dB) as a function of Great Circle Path distance from NWC to the LIE. 
field, calculated from the skin depth appropriate to the LIE. This modification has already been done and will be reported on in due course. R. Yeo has developed a 3D Born code that utilises the LWPM propagation facility, and also allows for $3 \mathrm{D}$ vector fields and source currents in the LIE. This powerful code will also shortly be modified with an attenuation function applied to the incident field. For short range scattering problems modal theory is less efficacious, and use of a full wave propagation code is called for. Such a version of the 3D Born code has yet to be constructed.

In conclusion, this work underlines the fact that many Trimpi scattering problems will violate the Born approximation and that intermodal scattering needs to be an integral part of any theoretical and modelling approach. We feel we have pointed the way towards an accurate and precise modelling of Trimpi scattering, a task made possible by recent increases in available computer power.

\section{Acknowledgements}

One author, Dr David Nunn, wishes gratefully to acknowledge his appointment to a Monbusho/British Council Visiting Professorship at the University of Electro Communications, in Chofu, Tokyo, Japan. The authors also wish to thank NOSC for the use of the MODEFNDR VLF propagation programme. This work has, in part, been supported by NASDA's Earthquake Remote Sensing Frontier Project.

\section{References}

Baba, K., Hayakawa, M., 1995. The effect of localised ionospheric perturbations on subionospheric VLF propagation on the basis of the finite element method. Radio Sci., 30 (5), 1511-1517.

Baba, K., Nunn, D., Hayakawa, M., 1998. The modelling to Trimpis on the path NWC-Dunedin using both finite element and 3D Born modelling. Geophys. Res. Lett., in press.

Baba, K., Hayakawa, M., 1996. Computational results of the effect of localised ionospheric perturbations on subionospheric VLF propagation. J. Geophys. Res. 101, 10,985$10,993$.

Bullough, K., 1988. Power Line Harmonic Radiation: Sources and environmental effects. In: Volland, H. (Ed.), Handbook of atmospheric electrodynamics, vol. 1, 2nd ed. CRC Press, Boca Raton, Florida, U.S.A., pp. 291-332.

Bullough, 1995

Clilverd, M., Yeo R.F., Nunn, D., Smith, A.J., 1998. Latitudinally dependent Trimpi effects: modelling and observations. Submitted to J. Geophys.

Dowden, R.L., Adams, C.D.D., 1989. Phase and amplitude perturbations on the NWC signal at Dunedin from lightning induced electron precipitation. J. Geophys. Res. 94, 497-503.

Dowden, R.L., Adams, C.D.D., 1990. Location of lightning induced electron precipitation from measurements of VLF phase and amplitude perturbations on spaced antennas and on two frequencies. J. Geophys. Res. 95, 4135-4145.

Dowden, R.L., Adams, C.D.D., Brundell, C.B., Dowden, P.E., 1994. Rapid onset, rapid decay (RORD) phase and amplitude perturbations on VLF subionospheric transmissions. J. Atmos. Terr. Phys. 52, 329-339.

Dowden, R.L., Brundell, J., Rodger, C., Molchanov, O.A., Lyons, W., Nelson, T., 1996. The structure of red sprites determined by VLF scattering. IEEE Antenna Prop. 38, 715.

Ferguson, J.A., Snyder, F.P., 1990. Computer programs for assessment of long-wavelength radio communications (Version 1.0: full Fortran code user's guide, 1 April 1990), NOSC Tech. Document 1773, National Ocean System Center, San Diego.

Helliwell, R.A., Katsufrakis, J.P., Trimpi, M.L., 1973. Whistler induced perturbations in VLF propagation. J. Geophys. Res. 78, 4679-4688.

Inan, U.S., Carpenter, D.L., 1987. Lightning induced electron precipitation events observed at $\mathrm{L}=2.4$ as phase and amplitude perturbations of subionospheric VLF signals. J. Geophys. Res. 92, 3106-3116.

Inan, U.S., Shafer, D.C., Yip, W.Y., Orville, R.E., 1988. Subionosperic VLF signatures of nighttime D region perturbations in the vicinity of lightning discharges. J. Geophys Res. 93, 11455-72.

Inan, U.S., 1990. VLF heating of the lower ionosphere. Geophys. Res. Lett. 17, 729-732.

Lev-Tov, S.J., Inan, U.S., Smith, A.J., Clilverd, M.A., 1996. Characteristics of localised ionospheric disturbances inferred from VLF measurements at two closely spaced receivers. J. Geophys. Res. 101, 15737-15747.

McDonald, B.H., Wexler, A., 1972. Finite element solutions of unbounded field problems, IEEE Trans. Microwave Theory Tech. 20, 841 .

Morfitt, D.G., Shellman, C.H., 1976, MODESRCH, an improved computer program for obtaining ELF/VLF mode constants. Interim report 77T, NTIS, ADA 032473, Naval Electronics Laboratory Center, U.S.A.

Molchanov, O.A., Parrot, M., 1995. PLHR emissions observed on satellites. J. Atmos. Terr. Phys. 57, 493-506.

Molchanov, P.A., Hayakawa, M., Ondoh, T., Kawai, E., 1998. Precursory effects in the subionospheric VLF signals for the Kobe earthquake. Physics of the Earth and Planetary Interiors $105,239-248$.

Molchanov, O.A., Shvets, A.V., Hayakawa, M., 1998. Analysis of lightning induced ionization from VLF Trimpi events. Submitted to J. Geophy. Res.

Nunn, D., 1997. On the numerical modelling of the VLF Trimpis effect. J. Atmos. Terr. Phys. 59, 537-560.

Poulsen, W.L., Inan, U.S., Bell, T.F., 1993. A multiple mode three dimensional model of VLF propagation in the Earth ionosphere waveguide in the presence of a localised $\mathrm{D}$ region disturbance. J. Geophys. Res. 98, 1705-1717.

Reagan, J.L., Meyeroff, R.E., Gunten, R.C., Imhof, W. L., Gaines, E. E., Larsen, T. R., 1982. Modeling of the ambient and disturbed ionospheric media pertinent to ELF/VLF propagation. AGARD Conf. Proc. 305. 
Schonland, B.F.J., Craib, M., 1927. Electric fields of S. African thunderstorms. Proc. Royal Society A 114, 229-243.

Simonich, D.M., Yeh, K.C., 1972. A theory of scattering from irregularities in a magnetoionic medium. Radio Sci. 17, 291299.

Smith, A.J., 1996. Aspects of wave particle interactions at mid latitudes. Adv. Space Res. 17 (10), 213-222.

Strangeways, H.J., 1996, Lightning, Trimpis and Sprites. Reviews of Radio Science, 1993-1996. Stone, R., (Ed.), Oxford University Press, Oxford, pp. 741-780.
Wait, J.R., 1964. On phase changes in VLF propagation induced by an ionospheric depression of finite extent. J. Geophys. Res. $69,441-446$.

Wait, 1964a

Wait, J.R., 1991. VLF radio wave mode conversion for ionospheric depressions. Radio Sci. 26, 1261-1265.

Wait, J.R., 1995. VLF scattering from a column of ionisation in the Earth ionosphere waveguide. J. Atmos. Phys. 57, 955-959. Williams, E.R., 1992. The Schumann resonance: A global tropical thermometer. Science Vol. No 1184-1188. 